\title{
SPEED-INDEPENDENT GAIT IDENTIFICATION FOR MOBILE DEVICES
}

\author{
GONZALO BAILADOR DEL POZO, CARMEN SÁNCHEZ-ÁVILA, \\ ALBERTO DE-SANTOS-SIERRA and JAVIER GUERRA-CASANOVA
}

\begin{abstract}
Due to the intensive use of mobile phones for different purposes, these devices usually contain confidential information which must not be accessed by another person apart from the owner of the device. Furthermore, the new generation phones commonly incorporate an accelerometer which may be used to capture the acceleration signals produced as a result of owner's gait. Nowadays, gait identification in basis of acceleration signals is being considered as a new biometric technique which allows blocking the device when another person is carrying it. Although distance based approaches as Euclidean distance or dynamic time warping have been applied to solve this identification problem, they show difficulties when dealing with gaits at different speeds. For this reason, in this paper, a method to extract an average template from instances of the gait at different velocities is presented. This method has been tested with the gait signals of 34 subjects while walking at different motion speeds (slow, normal and fast) and it has shown to improve the performance of Euclidean distance and classical dynamic time warping.
\end{abstract}

Keywords: Biometrics; gait; accelerometer; dynamic time warping.

\section{Introduction}

In biometrics, the term "gait" is used to describe a particular manner or style of walking which is distinctive for each individual. ${ }^{1}$ Although gait shows a common pattern for everybody, it also presents some interpersonal differences which make possible individual identification. This fact may be observed in our ability to recognize a person only by observing his/her gait.

In previous literature, different sensors have been used to capture the human gait. Many works ${ }^{2-4}$ proposed to extract the movement of lower limbs from images recorded by a camera. In other works, the authors ${ }^{5,6}$ extracted the movement of the 
legs by using some pressure sensors incorporated to the floor of the room. However, both approaches limit the acquisition to specific indoor environments. In order to alleviate this restriction, some authors used some wearable sensors which allow the subject to move freely. ${ }^{7}$ Due to the miniaturization of inertial sensors, some works have also proposed to use gyroscopes and accelerometers to measure these body movements. ${ }^{8,9}$ In particular for this paper, the human gait is captured by means of a tri-axial accelerometer.

In former works, the authors have placed an accelerometer on the back ${ }^{10,11}$ or on the chest ${ }^{12}$ in order to classify the different activities performed by a subject. Although these positions make it possible to differentiate between several activities, they are not adequate to identify subjects, as the movements captured in these positions are similar among most individuals. The first work that analyzed the acceleration of the gait as a biometric technique was performed by Ailisto and Mäntjärvi et al. in Ref. 8 . They performed an experiment over 36 subjects by placing the accelerometer at their waists. This is an interesting position since the accelerometer can be incorporated easily in the buckle of the belt. Another place that could be comfortable for users is their hip; however, signals captured at this place are not well-balanced since the sensor is closer to one leg in relation to the other one. Although other authors ${ }^{9,13}$ have tried to identify subjects from the accelerations of their ankles, capturing the movement of only one leg is a bit restricted since the movement of other body parts are also specific for each subject. The work presented in Ref. 14 shows that measuring the accelerations at several body parts considerably increases the identification performance, however this implies that the user must wear many sensors which could be uncomfortable. In our proposal, only one accelerometer was placed near the sacral vertebra since the center of gravity (COG) is close to this position. Therefore, the accelerations measured at this place represent a summary of the accelerations of the whole body. The COG is approximately at the same height of the waist so the sensor was attached to the belt of the subject.

Several techniques have been proposed to discriminate among gait acceleration signals of different individuals. In Ref. 9, the authors extracted the walking cycle length and some histogram statistics from the gait accelerations of each subject to perform this discrimination. The work presented in Ref. 8 compared the identification results obtained by these histogram statistics with two other features: the coefficients of Fast Fourier Transform calculated over a window of the acceleration signal and the correlation between the walking cycles and a template obtained during the enrolling time. This work concluded that the best result - an equal error rate of $7 \%$ - is achieved by the correlation method. Other works have also generated templates of the gait cycles from the data captured during the enrolment phase. In Refs. 13 and 15, the authors divided the enrolment signal into steps averaging all these steps to create a gait template. The amplitude and length of each step were normalized using linear interpolation in order to produce a template independent of the variations on speed and amplitude of the signals. Other authors have proposed to 
use a normalization based on dynamic time warping (DTW) since it is able to deal with nonlinear time variability. ${ }^{16}$ Nevertheless, in this paper they did not evaluate the system performance with gaits at different speeds.

Recent works in human gait ${ }^{17,18}$ have shown the high intra-variability present in the gait. In these studies, the authors affirm that the gait of the same person at different speeds can be as different as other person's gait. Furthermore, they also remark on the great differences in the gait of the same individual when using different kind of shoes.

In this paper, an experiment has been conducted to analyze the differences in gait identification performance when the walking speed varies considerably. In an initial phase, Euclidean distance and classical DTW algorithm were used to calculate the distances among gaits of different individuals at several speeds. Due to the fact that these algorithms have shown to be insufficient to deal with this speed variability, we have generated a gait template which summarizes the signals of gaits at different speeds. This template generation is based on the work presented in Ref. 19 where the authors generate templates for the recognition of upper limb gestures at different speeds.

The rest of the paper is structured as follows. First, we describe the acquisition of the acceleration signals and the preprocessing applied to them. After that we present how the experiments were performed to analyze the variability produced by the speed. Before describing the method which extracts the template from the gait at different speeds, we present the results obtained by Euclidean distance and classical DTW. Then, the results obtained by our template extraction technique are shown. Finally, we conclude the paper stating our final conclusions.

\section{Signal Capture}

The acceleration of the COG during a walk is captured with the tri-axial accelerometer sensor. This sensor was connected by a bluetooth connection to a personal digital assistant which stored the acceleration signals during the walks. The sensor was placed close to the COG of the person. The axes of the accelerometer were oriented so that the $X$-axis was pointing to the floor, the $Z$-axis to the left of the user and the $Y$-axis pointing forward. Using the biologic nomenclature, the axes are named: vertical $(X)$, anteroposterior $(Y)$ and mediolateral $(Z)$. The acquisition rate was fixed at $100 \mathrm{~Hz}$ which is sufficient since the frequencies of typical movement of the legs are usually lower than $12 \mathrm{~Hz}^{20}$ The range of accelerations measured by the sensor is $[-2,2] g$ because during the normal gait most accelerations of the waist are within this range.

Some previous works using an accelerometer placed at the $\mathrm{COG}^{21,22}$ have described that vertical and anteroposterior accelerations repeat a discernible pattern that consists of two quasi-sinusoidal signals. These signals are produced by the typical swinging of the pelvis in both directions during the gait cycle. Therefore, these sinusoidal signals present the same frequency though there is a phase-shift 


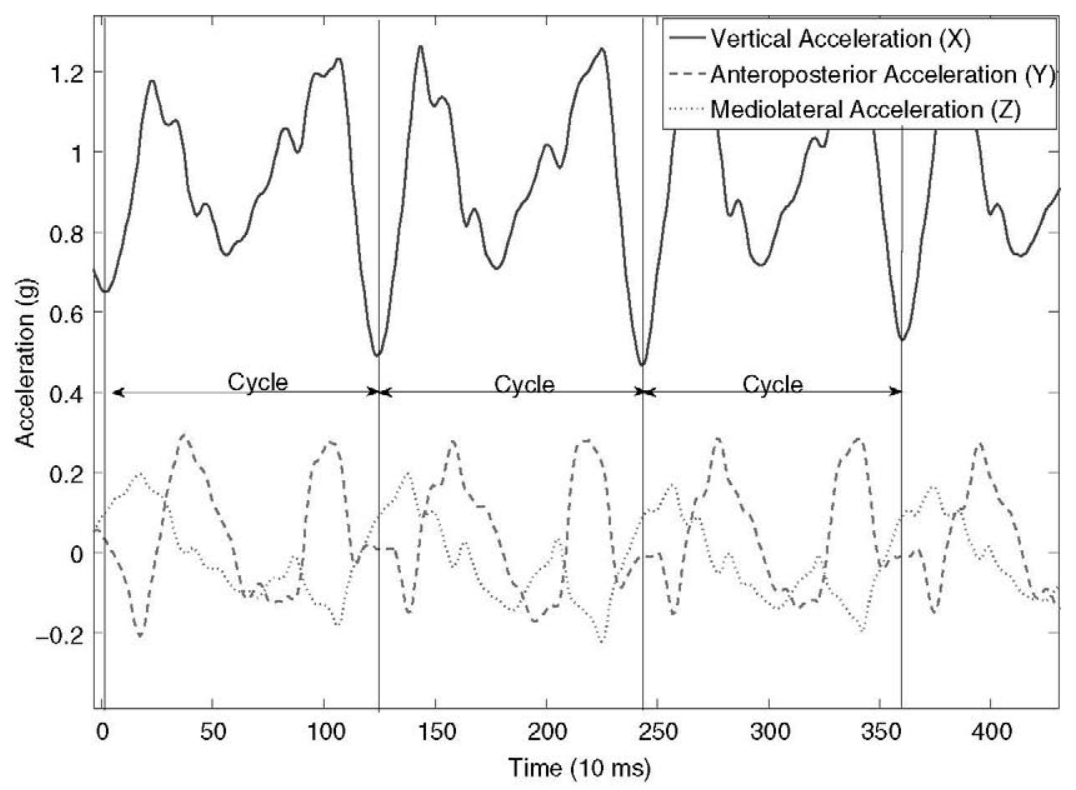

Fig. 1. Example of acceleration signals for several walking cycles.

between them. In the case of mediolateral acceleration, it presents a monophasic pattern since it depends on which limb is lifted. Furthermore, in contrast to the others accelerations, the authors of Ref. 23 have remarked the difficulty of finding a common pattern in this acceleration for all subjects. This means that the mediolateral acceleration is user-dependent so it may be crucial when identifying people. An example of these captured signals can be observed in Fig. 1 in which several cycle steps are depicted.

Although, in this work, we have manually segmented the signal in steps, some previous works show the feasibility of the automatic segmentation thanks to the sinusoidal behavior of vertical and anteroposterior accelerations. ${ }^{8,16}$ For instance, in Ref. 8 , the authors propose to detect the maxima in the vertical acceleration to segment into steps.

After segmenting the signal into steps, each step has been normalized in amplitude. On the one hand, the offset of the signal is removed to ignore the contribution of the gravity force whether the sensor is not completely well oriented. On the other hand, the signal is divided by the standard deviation of the signal since slight changes in the walking speed may produce high variations in the magnitude of the accelerations.

\section{Experiment Setup}

In this experiment, 34 subjects (23 male and 11 female) walked about 40 steps along a corridor. All these signals were segmented in gait cycles which correspond to two 


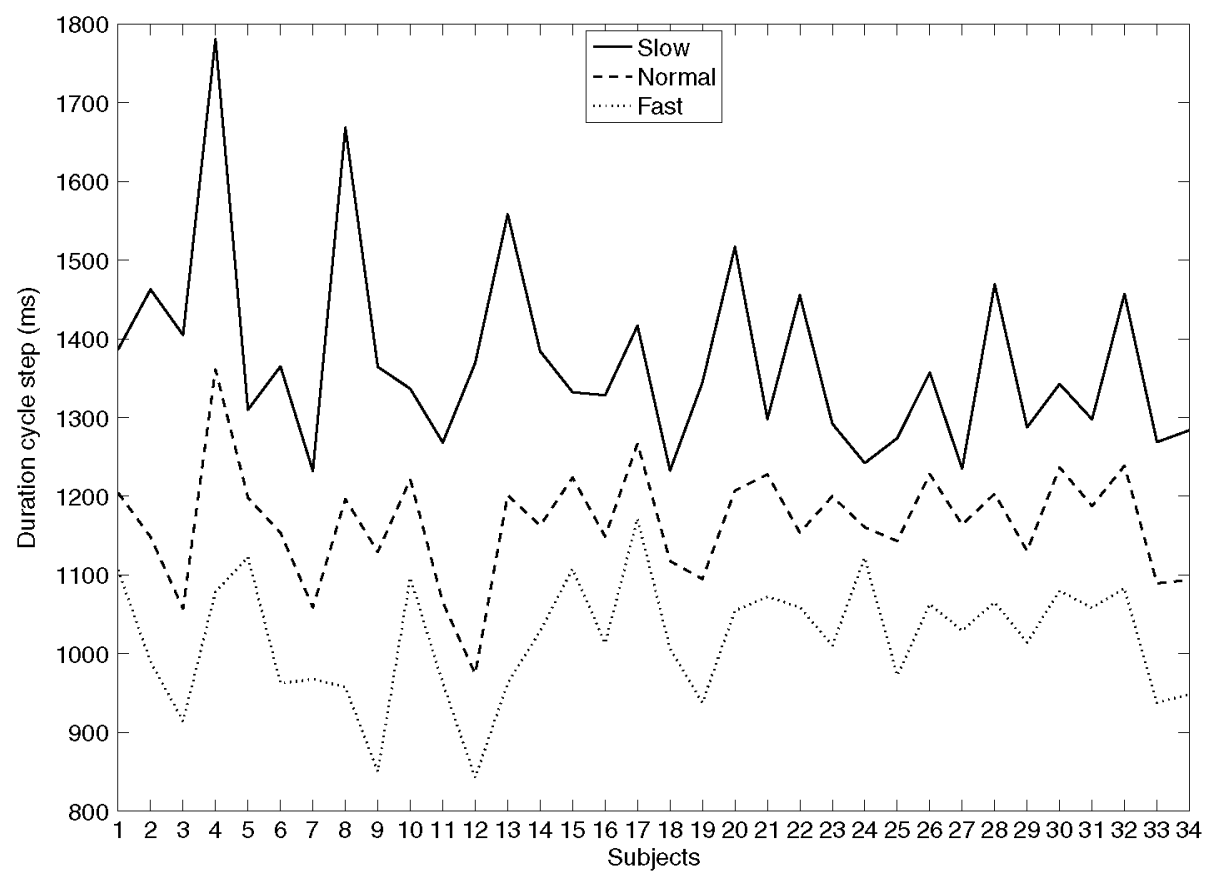

Fig. 2. Duration of the steps of the subjects at different speeds.

steps. This means that there are 20 gait cycles for each walk. Nevertheless, initial and end cycles were rejected because they are special cases. Hence, for each walk there are 18 segmented gait cycles.

The subjects repeated this walk three times at three different speeds: slow, normal and fast. In the slow speed, subjects were asked to walk quietly as they were thinking about something. The normal speed corresponds to their usual walking speed. Last, for the fast speed, subjects were asked to walk fast as they were arriving late to a meeting but without running. Therefore, overall, 1836 gait cycles ( 3 speeds $\times 34$ subjects $\times 18$ steps) have been captured.

The high variability in the length of steps at different speeds may be observed in Fig. 2 where the average durations of the steps for each individual and speed are represented.

\section{Euclidean Distance}

As an initial approach to discriminate the gaits of different subjects, we have calculated the distances between the acceleration signals of the segmented steps of each subject. Due to the fact that two steps of different subjects or at different speed may have different lengths, they must be rescaled in order to compare them. Some works have proposed to perform a global rescaling of the signals by means of interpolating techniques and then calculate the Euclidean distance since all signals present the 


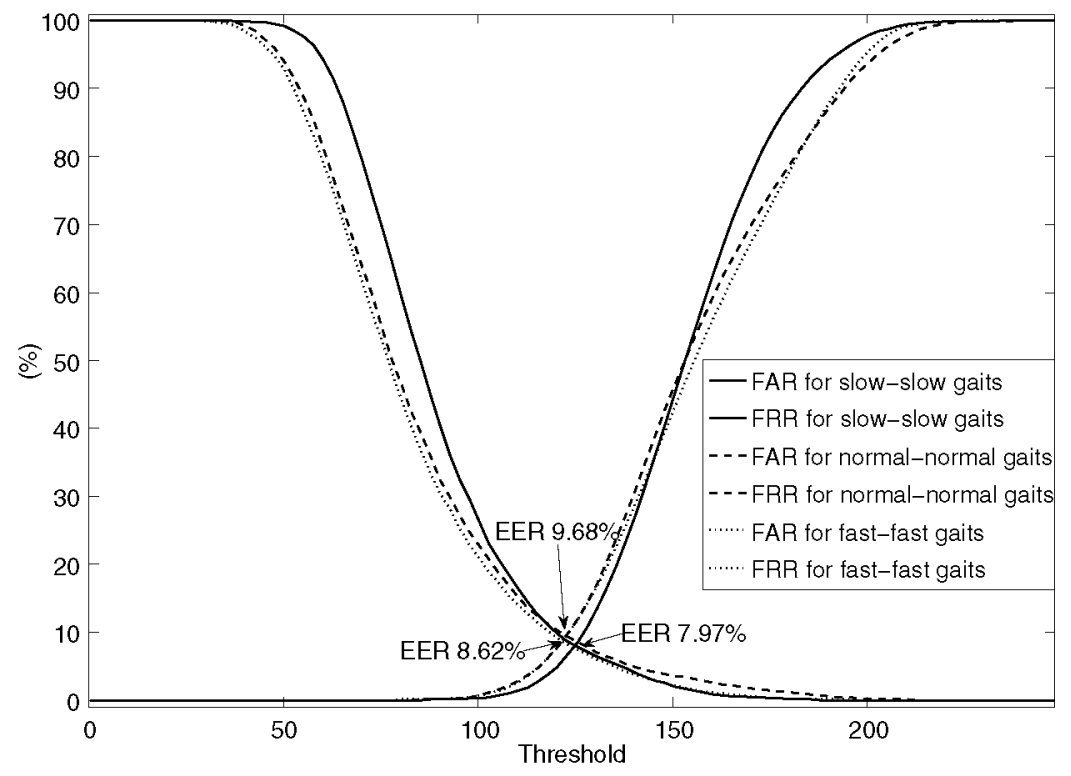

Fig. 3. FAR and FRR curves for Euclidean distance approach when comparing steps at the same speed.

same length. ${ }^{13,18}$ In this paper, all steps have been rescaled to a length of 100 samples using a polyphase filter implementation.

After that, we have analyzed the similarity among the gait signals of the same individual at the same speed and the differences when comparing them with the gait signals of other individuals. The results of this analysis have been depicted in Fig. 3 by means of three curves of false acceptance rate (FAR) and false rejection rate (FRR). Each curve represents the rates obtained for a specific speed (slow, normal and fast) when comparing the signals of different subjects at this same speed.

It can be observed that all equal error rates (EER) are close to $10 \%$ which is quite high. The reason of this poor performance could be that the global time rescaling is not adequate for this problem. Consequently, a DTW approach has been tested since it is able to align dynamically two signals.

\section{Dynamic Time Warping}

DTW algorithm generates a matrix $M$ with the costs of aligning the samples of two signals. In our case, the cost of each element $m_{i j}$ is the Euclidean distance between the 3D acceleration vectors of the points $i$ and $j$ of both compared instances, respectively. After that, the algorithm determines the path over this matrix which allows aligning both signals producing the minimum cost. This path $W$ is called "warping path" and represents how both signals must be extended or compressed in order to be aligned. 


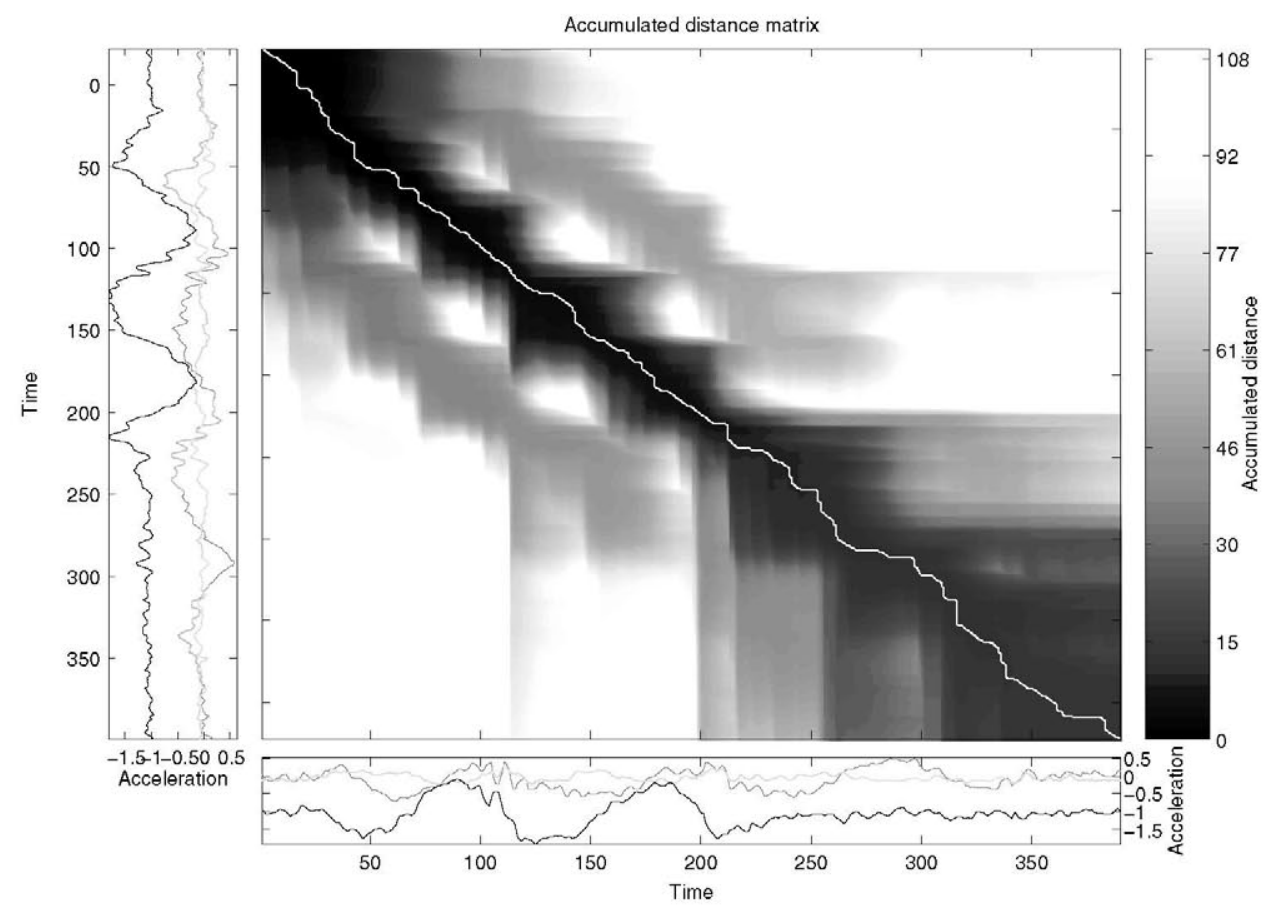

Fig. 4. Example of alignment using DTW.

Hence, the distance between two signals will be the sum of each element cost of the chosen warping path. This distance will be divided by the length of the warping path $W$ in order to make it independent of the length of the signals. An example of the DTW algorithm for two signals is represented in Fig. 4 where matrix $M$ is depicted with the accumulated costs and the warping path $W$ using a white line.

As can be observed in Fig. 5, the FAR and FRR curves when comparing gaits at the same speed show a significative improvement in comparison to Euclidean distance. An EER of $1.5 \%$ is obtained when comparing gait signals at fast speed whereas for slow and normal speeds EER is a bit higher, about $2 \%$. This difference in performance may be caused by the fact that the noise affects more the signals at low speed since their magnitude is comparable to the magnitude of noise.

In Fig. 6, the distances between gait signals at different speeds are compared. Although the EERs achieved when comparing gaits of similar speeds (slow-normal and normal-fast) have increased considerably, up to $14 \%$ and $12 \%$, respectively, the worst performance, about $32 \%$, has been reached when comparing gaits at quite different speeds (slow-fast). Therefore, these results indicate that gaits of the same individual at different speeds may produce signals different enough to be identified as belonging to other individual. 


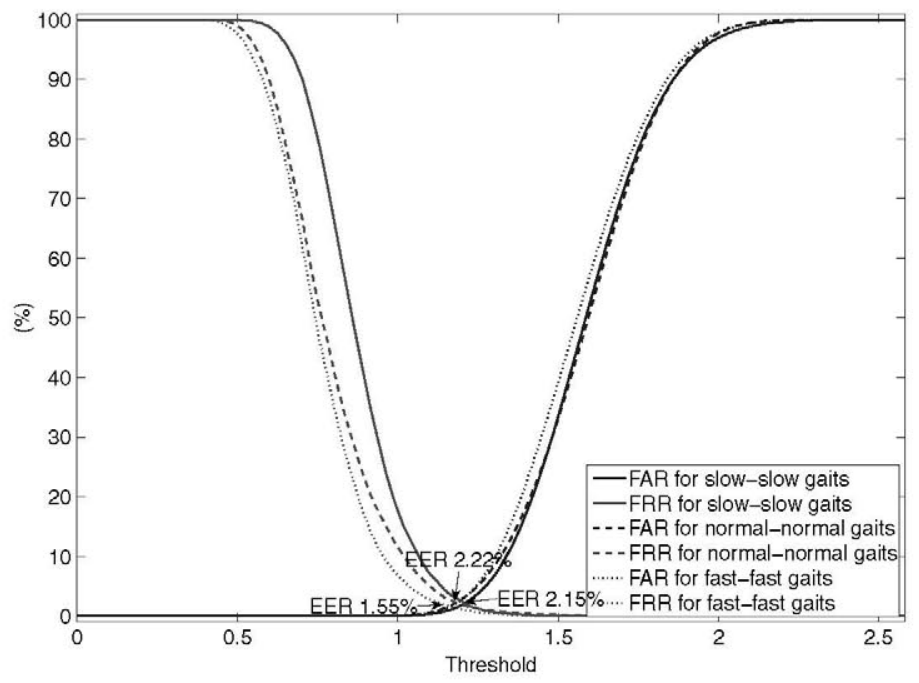

Fig. 5. FAR and FRR curves for classical DTW approach when comparing steps at the same speed.

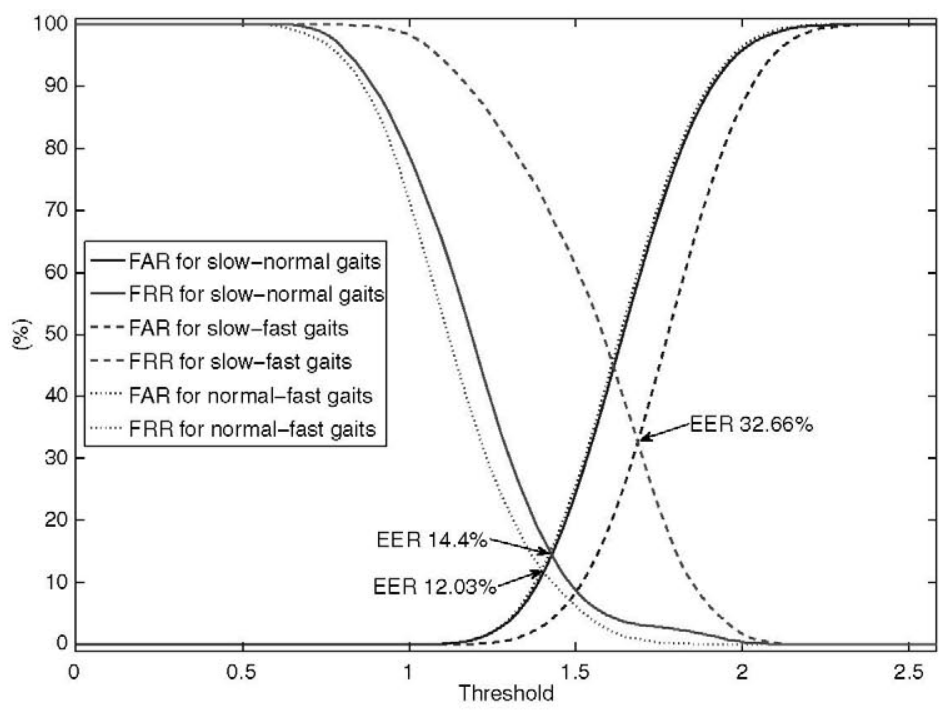

Fig. 6. FAR and FRR curves for classical DTW approach when comparing steps at different speeds.

Finally, we have represented, in Fig. 7 the FAR and FRR curves when ignoring the speed of the gait, i.e. we only distinguish between gaits of different individuals without taking into account their respective speeds. Hence, the EER of $19.31 \%$ shown in this figure may be considered as an overall measure of the performance of DTW to deal with gaits at different speeds. 


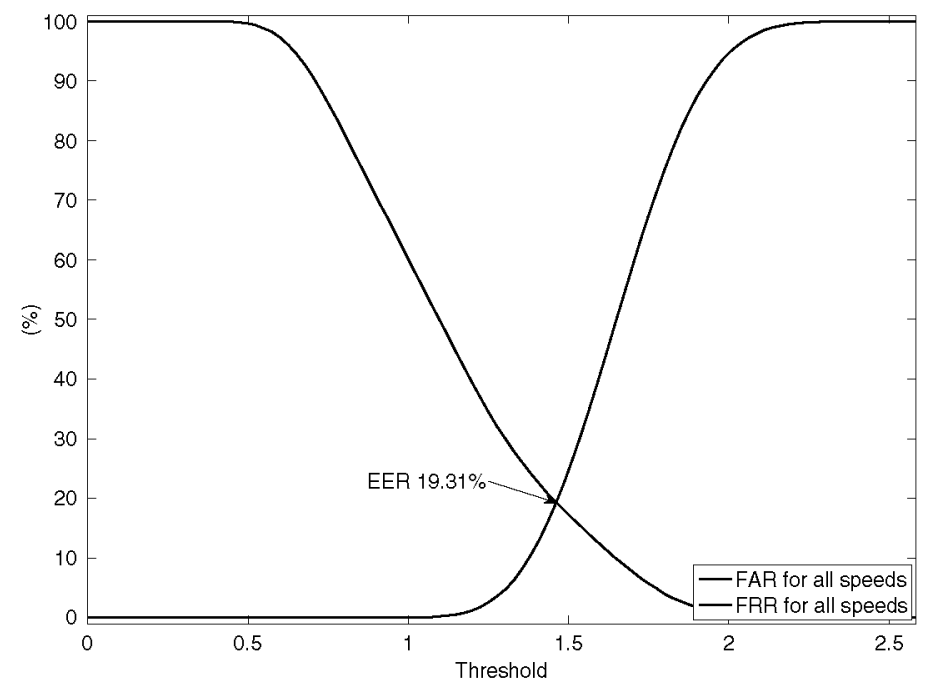

Fig. 7. FAR and FRR curves for classical DTW approach when all steps are compared without taking into account their speeds.

\section{Average Template DTW}

A solution to improve the poor results obtained when comparing gaits at different speeds could be the generation of a template for each subject using gait cycles at all speeds. Then the decision of the ownership of a gait signal will be based on the distances to this template. In this paper, we propose to extract an average template based on the work in gesture recognition presented in Ref. 19. Concretely, three gait instances at slow $I_{S}$, normal $I_{N}$ and fast $I_{F}$ speed have been used to generate a template of the gait for each individual. These instances have been randomly selected from the 18 available instances for each subject and speed. The rest of samples ( $51=3$ speeds $\times 17$ samples) have been used to test the performance of the system. This selection has been repeated 10 times in order to take into account the variability of different samples.

The template extraction of our approach uses an algorithm based on DTW to align the gait signals at different speeds. Similar solutions have been proposed in Refs. 16 and 19 however, these methods attempted to align independently the gait signal for each dimension $(X, Y, Z)$ and to fuse subsequently the information of all three dimensions. Our alignment algorithm considers the gait signal as a collection of $3 \mathrm{D}$ points and therefore the distance in $3 \mathrm{D}$ space measures the closeness between samples. In the following paragraphs, the alignment algorithm is explained.

For each person, the first step of this algorithm is to calculate the DTW distances between all possible pairs of all gait instances $\left(I_{S} I_{N}, I_{N} I_{F}\right.$ and $\left.I_{S} I_{F}\right)$ in order to decide what instance will be used as a reference. The reference instance $I_{R}$ will be the one that presents the smallest average distance to all the remaining instances. We 
consider that $I_{R}$ is normalized and the remaining instances are normalized using their warping paths respect to this reference instance. Each warping path $W$ represents how to compress and expand the time scale of the given instance $I$ to minimize the distance with $I_{R}$. Between every pair of consecutive elements $w_{k}$ and $w_{k+1}$ of the warping path $W$ (see Fig. 4), the DTW algorithm allows only three types of movement along the matrix $\mathrm{M}$ : horizontal, vertical and diagonal. If this movement is horizontal the samples $I(t)$ and $I(t+1)$ of the given instance $I$ are compressed and whether it is horizontal the sample $I(t)$ is extended to the sample $I(t+1)$. In the case that the movement is diagonal, no rescaling is applied.

Compressing samples means joining them and this produces a loss in the original information. In order to minimize this loss, these points are combined by calculating their average. In the case of extending a sample, the original value of the sample is repeated for the next samples. After rescaling all gait instances, for each instance a normalized sequence is obtained which has the same length as the reference instance $I_{R}$. At this point, we propose to extract the average template by calculating the average points for each time sample. An example of this template extraction is shown in Fig. 8, where the average template is obtained from slow, normal and fast gait signals.

Figure 9 depicts the results obtained when the average templates are compared to the gait signals of all different speeds at the same time. Due to the fact that each experiment has been performed 10 times in order to obtain results independent from the chosen instances, the FAR and FRR curves shown in this figure represents the average curves for all ten trials. Furthermore, in order to show the variability of the results, the standard deviation is also represented by means of some dotted lines
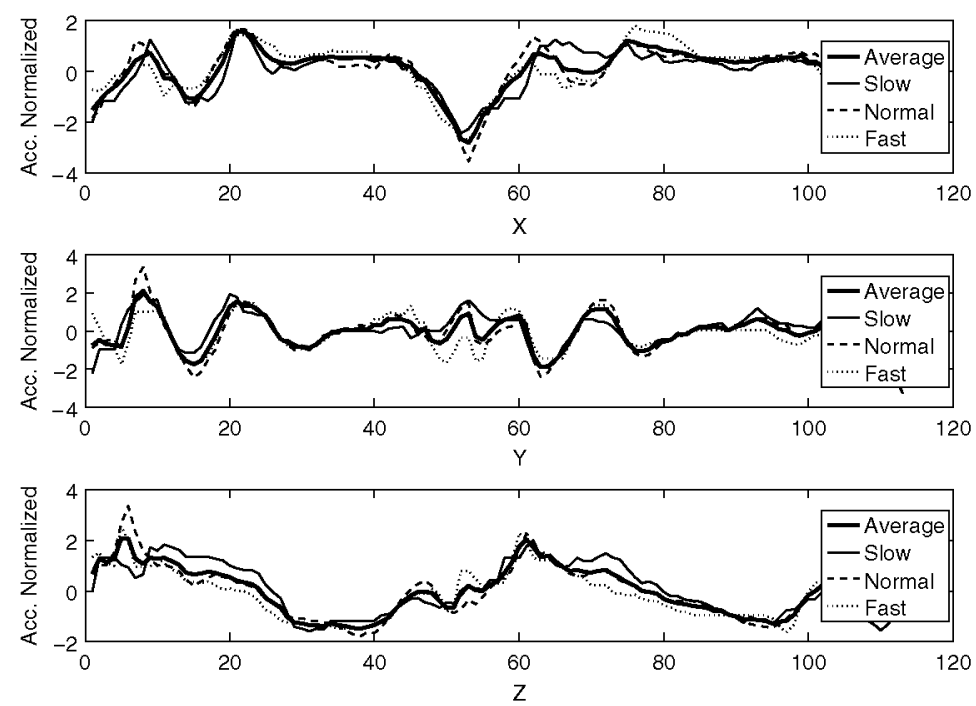

Fig. 8. Example of template extraction. 


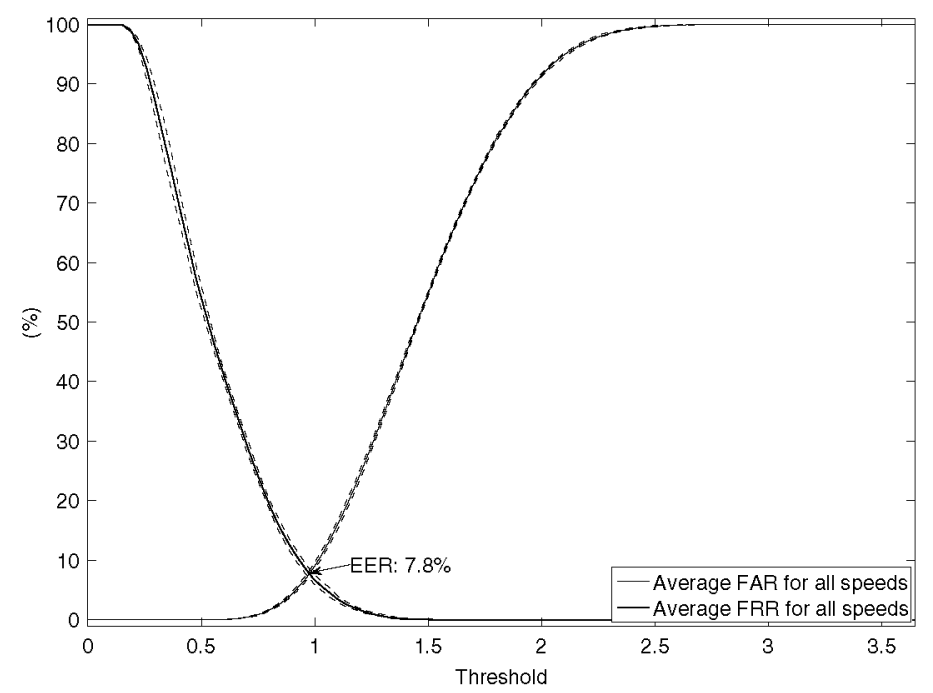

Fig. 9. FAR and FRR curves for average template DTW approach when all steps are compared without taking into account their speeds.

surrounding the FAR and FRR average curves. The average EER is about $7.81 \% \pm$ $0.5 \%$ which is much lower than the obtained by classical DTW $19.31 \%$.

\section{Conclusion}

In this paper, we have concluded that Euclidean distance and dynamic time warping are not able to deal properly with changes in the gait signal caused by different walking speeds. Nevertheless, the extraction of a template from gaits at different speeds has shown to improve the identification overall performance from $19.31 \%$ to $7.81 \%$ which makes this technique appropriate to noncritical security problems. Furthermore, the use of only one template for modeling the individual gait supposes to perform only one comparison when identifying the gait signal of a user saving a considerable amount of time. Therefore, the identification process has not a high computational cost which makes it suitable to devices with low computational power as smartphones or other mobile devices. This feature is specially interesting since one of the main applications of the gait identification based on accelerometer signals is to detect whether a mobile device has been stolen by detecting changes in the gait signals.

\section{References}

1. T. Kobayashi and N. Otsu, Three-way auto-correlation approach to motion recognition, Pattern Recog. Lett. 30(3) (2009) 212-221. 
2. C. BenAbdelkader, R. Cutler, H. Nanda and L. Davis, Eigengait: Motion-based recognition of people using image self-similarity, in Audio- and Video-Based Biometric Person Authentication (Halmstad, Sweden, 2001), pp. 284-294.

3. D. Cunado, M. S. Nixon and J. N. Carter, Automatic extraction and description of human gait models for recognition purposes, Comput. Vis. Image Understanding 90(1) (2003) $1-41$.

4. M. S. Nixon, T. Tan and R. Chellappa, Human Identification Based on Gait (SpringerVerlag, New York Inc., 2006).

5. L. Middleton, A. A. Buss, A. I. Bazin and M. S. Nixon, A floor sensor system for gait recognition, in Fourth IEEE Workshop on Automatic Identification Advanced Technologies (Buffalo, New York, USA, 2005).

6. J. Jenkins and C. Ellis, Using ground reaction forces from gait analysis: Body mass as a weak biometric, Pervasive Computing (Toronto, Canada, 2007), pp. 251-267.

7. T. Liu, Y. Inoue and K. Shibata, New method for assessment of gait variability based on wearable ground reaction force sensor, in 30th Annual Int. Conf. IEEE on Engineering in Medicine and Biology Society, 2008, pp. 2341-2344.

8. H. J. Ailisto, M. Lindholm, J. Mantyjarvi, E. Vildjiounaite and S. M. Makela, Identifying people from gait pattern with accelerometers, in Proc. SPIE, Vol. 5779, 2005, p. 7.

9. D. Gafurov, E. Snekkenes and T. Buvarp, Robustness of biometric gait authentication against impersonation attack, in On the Move to Meaningful Internet Systems: OTM Workshops (Montpellier, France, 2006), pp. 479-488.

10. M. Sekine, T. Tamura, T. Fujimoto and Y. Fukui, Classification of walking pattern using acceleration waveform in elderly people, in 22nd Annual Int. Conf. IEEE on Engineering in Medicine and Biology Society, Vol. 2 (Chicago, Illinois, USA, 2000).

11. S. H. Lee, H. D. Park, S. Y. Hong, K. J. Lee and Y. H. Kim, A study on the activity classification using a triaxial accelerometer, in 25th Annual Int. Conf. IEEE on Engineering in Medicine and Biology Society, Vol. 3 (Cancun, Mexico, 2003), pp. 2941-2943.

12. S. Tanaka, K. Motoi, M. Nogawa and K. Yamakoshi, A new portable device for ambulatory monitoring of human posture and walking velocity using miniature accelerometers and gyroscope, in 26th Annual Int. Conf. Engineering in Medicine and Biology Society, EMBC 2004, Vol. 3 (San Francisco, California, USA, 2004), pp. 2283-2286.

13. D. Gafurov, E. Snekkenes and P. Bours, Improved gait recognition performance using cycle matching, Int. Conf. Advanced Information Networking and Applications Workshops (Perth, Australia, 2010), pp. 836-841.

14. G. Pan, Y. Zhang and Z. Wu, Accelerometer-based gait recognition via voting by signature points, Electron. Lett. 45(22) (2009) 1116-1118.

15. D. Gafurov, E. Snekkenes and P. Bours, Spoof attacks on gait authentication system, IEEE Trans. Inf. Forensics Security 2(3) (2007) 491-502.

16. L. Rong, D. Zhiguo, Z. Jianzhong and L. Ming, Identification of individual walking patterns using gait acceleration, in the 1st Int. Conference Bioinformatics and Biomedical Engineering, (ICBBE) (Beijing, China, 2007), pp. 543-546.

17. M. Bächlin, J. Schumm, D. Roggen and G. Tröster, Quantifying gait similarity: User authentication and real-world challenge, in M. Tistarelli and M. S. Nixon (eds.), Advances in Biometrics, Vol. 5558, Ch. 105 (Springer, Berlin, Heidelberg, 2009).

18. D. Gafurov and E. Snekkenes, Gait recognition using wearable motion recording sensors, EURASIP J. Adv. Signal Process. 2009 (2009) 1-16.

19. R. Muscillo, M. Schmid and S. Conforto, The Median Point DTW Template to Classify Upper Limb Gestures at Different Speeds, in 4th European Conf. Int. Federation for Medical and Biological Engineering, Springer, Antwerp, Belgium, pp. 63-66. 
20. C. Verplaetse, Inertial proprioceptive devices: Self-motion-sensing toys and tools, $I B M$ Syst. J. 35(3) (1996) 639-650.

21. J. J. Kavanagh, R. S. Barrett and S. Morrison, Upper body accelerations during walking in healthy young and elderly men, Gait Posture 20(3) (2004) 291-298.

22. M. Kourogi and T. Kurata, Personal positioning based on walking locomotion analysis with self-contained sensors and a wearable camera, in 2nd IEEE and ACM Int. Symp. Mixed and Augmented Reality ISMAR '03, IEEE Computer Society, Washington, DC, USA, 2003.

23. B. Auvinet, G. Berrut, C. Touzard, L. Moutel, N. Collet, D. Chaleil and E. Barrey, Reference data for normal subjects obtained with an accelerometric device, Gait Posture 16(2) (2002) 124-134.

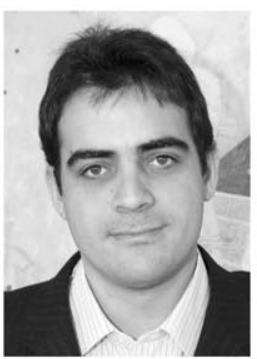

Gonzalo Bailador del Pozo obtained his Ph.D. in Computer Science in 2011, from Universidad Politécnica de Madrid (UPM). He is currently a postdoc researcher at CeDInt. He is an expert in pattern recognition methods and Soft Computing techniques. He has been a visiting researcher in ETH University and the European Centre of Soft Computing. His research lines cover topics related to movement analysis, biometrics and mobile applications.

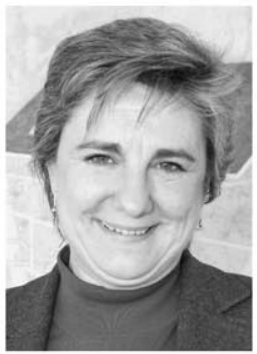

Carmen Sánchez Ávila received her Ph.D. in Mathematics Science from the Complutense University of Madrid, Madrid, Spain, in 1993. Since 1985, she has been with the Applied Mathematics Department, Polytechnical University of Madrid, Madrid, where she is currently a Professor and lectures on different subjects related to mathematics and numerical analysis, together with subjects covering topics in wavelets and biometric systems in Ph.D. courses. Her research lines cover topics related to signal processing, cryptography, and biometrics.

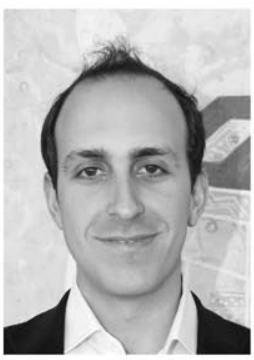

Alberto de-Santos Sierra was born in Madrid, Spain. He received his M.S. degree in Telecommunication Engineering from the Polytechnical University of Madrid, Madrid, in 2007, where he is currently working toward his Ph.D. with his doctoral thesis based on topics related to hand biometrics in mobile devices.

His research interests cover stress detection by means of physiological signals and iris biometrics.

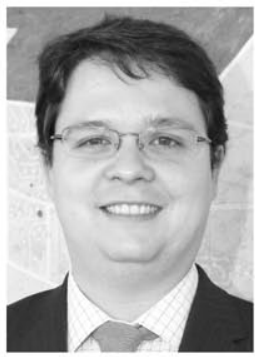

Javier Guerra Casanova was born in Madrid, Spain. He received his M.S. degree in Telecommunication Engineering from the Polytechnical University of Madrid, Madrid, in 2008, where he is currently working toward his Ph.D., with his doctoral thesis related to biometric identification by means of inair signatures carried out with devices embedding an accelerometer. 